\title{
EL COMPENDIUM DEL PADRE BARTOLOMÉ BRAVO
}

\author{
Antonia Maria Medina Guerra \\ Universidad de Málaga \\ ammedina@uma.es
}

\section{Resumen}

El diccionario latín-español de Bartolomé Bravo, el Compendium Marii Nizolii (Valladolid, 1619), se inspira, como puede deducirse de su título, en el repertorio de Nizzoli, pero además de esta obra el jesuita segoviano debió contar con el diccionario de Ambrogio Calepino o con una edición plurilingüe del Nizzoli, inspirada en el Dictionarium del lexicógrafo bergamasco. En este sentido, no podemos olvidar que la parte española del diccionario de Calepino tiene mucho que ver con la obra de Antonio de Nebrija y que, por tanto, Bravo se nos presenta en última instancia como un eslabón más de la cadena lexicográfica iniciada por el sevillano un siglo antes.

Al padre Bartolomé Bravo le debemos dos repertorios lexicográficos, uno español-latín, el Thesaurus verborum ac phrasium ad orationem ex Hispana Latinam efficiendam \& locupletandam (Zaragoza, 1597), y otro latín-español, el Compendivm Marii Nizolii sive Thesauri Marci Tvlii Ciceronis, quo tum verba tum varioe cuiur que formula, ac locutiones, quibus idem vf us ef t, continentur, Hispano idiomate $f$ ingulis verbis ad f cripto (Valladolid, 1619). Ambos diccionarios están destinados a los jóvenes que se inician en los estudios de la lengua latina, por lo que podría pensarse que el jesuita se limitó a un simple trasvase de información de uno a otro, a una tarea puramente mecánica. Para comprobarlo he cotejado las equivalencias de los artículos de la combinación $a b$ del Thesaurus con las entradas que se catalogan en el Compendium. Evidentemente, en esta comparación se aprecia, como es lógico al ser obras del mismo autor, muchas coincidencias, por ejemplo, las siguientes:

- Abatir a alguno. Deprimo, is [...].

* Deprimo, is, efsi, effum. Abatir, echar a fondo [...].

- Abaxo, o hazia abaxo. Deorfum [...].

* Deorrum. hazia abaxo [...].

- Abertura de la boca quando alguno re rie. Rictus, us.

* Rictus, us. Abertura de la boca.

- Ablandar. Mollio, is.

* Mollio, is. iui. itum. Ablandar [...].

- Abogacía. Officio de abogado. Aduocatio, onis. aduocati officum.

* Aduocatio, onis. Abogacía, oficio de abogado. 
- Aborrecer. Odi, odifti.

* Odi. odir ti. Aborrecer.

Sin embargo, también son muy numerosas las discrepancias, entre ellas y a modo de ejemplo se pueden señalar:

1. En la macroestructura del Compendium no se hallan muchos de los equivalentes latinos de las entradas españolas del Thesaurus. Así, abbas, tis; abbati $\Gamma$ a, a; abbatia, $\mathscr{x}$; af $\int$ uef co, is; patrocinior, aris; genealogia, a ; aquarium, ij; tribulus, $i$ o abania, ce no figuran en la nomenclatura del diccionario latino-español.

2. En bastantes ocasiones, los equivalentes latinos del Thesaurus se recogen en el Compendium con otro sentido, o lo que es lo mismo, no siempre las entradas españolas del Thesaurus coinciden con las traducciones de las entradas latinas del Compendium':

- Abominación. Execratio, onis.

* Execratio, onis. Maldición [...].

- Abrir puerta. Patefacio, is.

* Patefacio, is. penultima correcta feci, actum. Def cubrir, declarar [...].

- Abroluer. Abroluo, is.

* Abroluo, is, vi, utum. Acabar. Perfeccionar [...].

Además, el número de entradas del Thesaurus es superior al del Compendium². Así, por ejemplo, muchos de los términos de carácter religioso que aparecen en el Thesaurus no se hallan en el Compendium. Estas voces, como es obvio, no son propias de los escritos ciceronianos, principal fuente de la obra de Nizzoli, en la que está inspirada el Compendium. Este hecho viene a respaldar la hipótesis de que el Thesaurus y el Compendium toman como referencia los diccionarios de Nebrija y Nizzoli, respectivamente (Medina Guerra, 1997).

En cualquier caso, ambos repertorios gozaron de un gran éxito y prestigio, contando con un buen número de ediciones hasta bien entrado el siglo XIX, aunque las del Thesaurus superan con creces a las del Compendium ${ }^{3}$. Este último -diccionario al que le dedicaré las páginas que siguen- no llegó a verlo impreso su autor, pues sale a la luz por primera vez en

1 Es más, a diferencia del Dictionario de vocablos castellanos aplicados a la propiedad latina de Sánchez de la Ballesta, de la macroestructura del Thesaurus están ausentes las entradas encabezadas por la voz cosa o las construcciones de relativo, que sí aparecen en la microestructura del Compendium (Inerans, atis; Lector, oris; Municipalis, e; Naualis, e; Politicus, a, um; RoItratus, a, um; Speculator, oris).

2 En el Thesaurus se catalogan más de 18000 entradas, mientras que en el Compendium se recogen en torno a las 14000 .

3 Según mis datos, el Thesaurus se publicó en más de 100 ocasiones, mientras que el Compendium contó con unas 50 ediciones. 
los talleres de Jerónimo de Murillo en $1619^{4}$, doce años después del fallecimiento del jesui$\mathrm{ta}^{5}$, gracias al empeño de su sobrino Pedro Bravo, según se indica en el "Privilegio Real":

Por qua[n]to por parte de vos Pedio Brauo Religioso de la $\mathrm{Co}[\mathrm{m}]$ pañia de lesus, nos fue fecha relacio[n], q[ue] por nos se auia dado lice[n]cia al Padre Bartolome Brauo, vuestro tio, difunto Religioso q[ue] fue de la dicha Co[m]pañia, para q[ue] pudiesse imprimir vn libro por el co[m]puesto, intitulado, Co[m]pendiu [m] M. Nizolii, siue Thesauri Ciceronis, y priuilegio por diez años, y por auer muerto el suso dicho no auia podido tener efecto la dicha impression, y del dicho libro originalmente ante los del nuestro Consejo hizistes presentar, rubricado de Cristoual Nuñez de Leon, escriuano de Camara, que fue del nuestro Consejo, y nos pedistes, $\mathrm{y}$ suplicastes mandaremos se os prorrogase, $\mathrm{y} \operatorname{co}[\mathrm{n}] \mathrm{cediesse}$ de nueuo priuilegio, para por tiempo de los diez años vos, y no otro ninguno pudiessedes imprimir, y vender el dicho libro sin incurrir en pena alguna, o como la nuestra merced fuesse $[\ldots]^{6}$.

El Compendium, al igual que el Thesaurus, está dirigido, como ya se ha dicho, a los estudiantes de latín. Bartolomé Bravo conoce como profesor ${ }^{7}$ la necesidad de utilizar en el aula obras que contribuyan a difundir la elegancia y pureza de la lengua latina entre la juventud. En el prólogo del Compendium exalta la labor realizada, entre otros, por Robert Estienne, Ambrogio Calepino o Mario Nizzoli, si bien reconoce que sus frutos han sido mayores en el resto de Europa que en España y que los repertorios de estos autores, fundamentales para el estudio de la lengua latina, son incómodos de manejar debido a sus grandes dimensiones. Por tanto, su objetivo será realizar una obra de tamaño más reducido que encierre lo mejor del latín. Con este fin, según se desprende ya del título y se deja claro en el prólogo $0^{8}$, toma como punto de partida el voluminoso Compendium de Mario Nizzoli del que pretende seleccionar únicamente lo esencial", traduciéndolo al español para hacerlo más accesible a los jóvenes. Esta reducción se hace evidente en el formato: todas las ediciones del Compendium se publican en $4^{\circ}$ y suelen medir en torno a los veinte centímetros, se trata, en consecuencia, de unas dimensiones acordes con la utilidad práctica perseguida por su autor. En concreto, la edición prínceps consta de 4 h. s. n., 449 págs., 64 págs., que se distribuyen como sigue:

Portada.- v. b.- Tasa (4 maravedís el pliego). Firmada en Madrid el 2 de octubre de 1619 por Lázaro Ríos.- "Correctiones erratorum". Firmada en Valladolid el 11 de agosto de 1619 por el Dr. Vergara Baraona.- "Licencia". Firmada en Valladolid el 2 de mayo de 1619 por Diego de Sousa.- Privilegio real.- Prefacio latino de Bartolomé Bravo.- Texto a

4 De esta primera edición se conservan ejemplares en la Biblioteca de la Facultad de Filología de la Universidad Complutense (30187 y 30188 ) y en la Biblioteca Universitaria de Sevilla (148/60); no obstante, me ha sido más fácil acceder al ejemplar de la edición vallisoletana de 1641 conservado en la Biblioteca Nacional de Madrid con la signatura $2 / 14910$ y es, por tanto, del que tomo los ejemplos que transcribo en estas páginas.

5 Atendiendo a las breves referencias biográficas recogidas por Tomás Baeza y González (1877: 136) o Gabriel María Vergara y Martín (1903: 455), murió en Medina del Campo (Valladolid) en 1607.

6 Transcripción del ejemplar conservado en la Biblioteca Universitaria de Sevilla.

7 Fue profesor de filología y gramática en la Universidad de Salamanca durante veintisiete años. C. M. ${ }^{a}$ Ajo González y Sáinz de Zúñiga lo cita como profesor del claustro de la universidad salmantina durante el reinado de Felipe II (1958: t. II, 248).

8 Bravo se distancia así de una práctica muy habitual entre los lexicógrafos españoles: la de no revelar sus fuentes. Véanse a este respecto trabajos como los L. Cooper (1962) o M. Alvar Ezquerra (1990: 55).

9 Así, por ejemplo, al comparar ambos repertorios se aprecia en el jesuita español una tendencia a eliminar los derivados de los verbos regulares. 
2 col. Encabezamiento: Compendivm Nizolii, sive Thesavri verborvm ac phasivm, quibus Marcus Tullius Cicero vsus est [449 págs.].- Breve vocabulario de voces tomadas de otros autores latinos [64 págs.].

La edición de 1641 (impresa también en Valladolid por Jerónimo Murillo), que es, como ya he indicado, la que tomo como referencia, contiene 3 h. s. n., 1 h. [grabado], 452 págs., 55 págs., $1 \mathrm{~h}$. [escudo de la Compañía de Jesús]:

Portada.- v. b.- "Suma de licencia" (Licencia al Colegio de San Ambrosio para imprimirlo por una vez).- "Fee de erratas". Firmada por Fr. Raphael Manso. San Pablo de Valladolid, 6 de febrero de 1641. - "Suma de la Tassa" (4 maravedís por pliego.- Prefacio latino de Bartolomé Bravo.- Texto a 2 col. Encabezamiento: Compendivm Nizolii, sive Thesavri verborvm ac phasivm, qvibus Marcus Tullius Cicero vsus est [452 págs.].- Breve vocabulario de voces tomadas de otros autores latinos [55 págs.].- Escudo de la Compañía de Jesús.

Sin embargo, a partir de la edición de 1671 [1670 $]^{10}$, corregida y adicionada por el también jesuita Pedro de Salas bajo el título Compendivm latino-hispanum utriusque linguae, el diccionario, aunque conserva su formato original en $4 .^{\circ}$, sufre importantes transformaciones en la macroestructura y en la microestructura -en las que no voy a detenerme en esta ocasión (Medina Guerra, 1998b)- que trae consigo un considerable aumento de volumen, superando con creces el millar de páginas ${ }^{11}$. Así, el ejemplar conservado en la Biblioteca Universitaria de Sevilla consta de 4 h. s. n., 1040 págs., 78 págs.

[mútilo de portada].- "Auiso para los que huuieren de aprobecharse de este compendio".- "A la muy noble, y muy leal ciudad de Valladolid. El Colegio de la Compañia de Iesvs de S. Ambrosio"'2. "Con licencias de los superiores del Ordinario y Supremo

10 A. Palau (1966: t. xvıII, n. 286149) cita la edición vallisoletana de 1671 impresa en "Ex Off. Bartholomaei Portoles et Torres" como la primera corregida y adicionada por Pedro de Salas. Es probable que el ejemplar conservado en la Biblioteca Universitaria de Sevilla con la signatura 9/55, mútilo de portada y catalogado de acuerdo con la "Tasa" como impreso en Madrid en 1670, corresponda a esta edición.

11 No deja de ser significativo que, a pesar de estas mejoras, este diccionario latino-español se vuelva a publicar con el título original y tal como lo concibió Bravo al menos en tres ocasiones más (Alcalá de Henares, "Apud Franciscum Garcia Fernandez Typographum Vniversitatis. A costa de Iuan Vicente Mercader de libros frontero de S. Felipe", 1672 [ejemplares en la Biblioteca Nacional de Madrid: 2/38021 y 2/46581]; Valladolid, "Ex Officina Iacobi Folquerol, Typographi Regii", 1676 [referencia tomada de Palau, II, n..$^{\circ} 34642$ ] y Burgos, "En la imprenta de Juan de Viar", 1682 [en el Biblioteca de Filología de la Universidad Complutense con la signatura 19350]).

12 Al igual que Bartolomé Bravo, Salas tampoco llegó a ver impresa la obra, según se indica en la dedicatoria: "El dedicarse esta obra â V. S. es obsequio debido en consecuencia de lo que obrô en vida cl Autor de ella; pues todas sus obras se dedicaron $\hat{a}$ V. S. y se hizieron en sus seruicio, consagrandose enteramente al bien de esta Republica, al vniuersal prouecho de sus almas, y â la particular Doctrina, y buena educacion de sus hijos. No es acordar â V. S. con la oferta presente lo que tan viuo tiene en la memoria: solo es mostrar, que el Autor no es otro en la muerte de lo que fue en su vida. Atenta la fama, que alcanço quando viuia, no se estrañara la correspondencia de que como justo sea el mismo, difunto, que viuo [...]. Siruio â V. S. el P. Pedro de Salas en la buena educacion de sus hijos, y fueron tantos los que su enseñança aprouecharon en virtud y letras, que me parece su numero compararse â las estrellas, si yâ no son estrellas del Cielo, que deuen â este Padre los rayos, con que ilustran el firmamento de la Iglesia [...]. De las muchas y eruditas obras que saco a luz, esta [es] la vltima, no solo en tiempo, sino en la perfeccion: porque â juyzio de la docta discrecion, no parece, que ay mas que hazer para la lengua Castellana, subiendo de quintales, llegue al mas alto punto de la elegancia Latina, y para que la perfeccion desta se haga assequible de todos con facilidad: y assi despues esta obra no hizo mas, porque no auia mas que hazer $[\ldots] "$. 
Consejo de Castilla".- Tasa (6 maravedís el pliego). Dada en Madrid el 23 de diciembre de 1670.- Erratas [sin firma de corrector].- Texto a 2 cols. [sin encabezamiento, 1040 págs.].- Lista de voces sacras.- Lista de voces hebreas [78 págs., en la pág. 65 comienza la Lista de voces hebreas] ${ }^{13}$.

Pero con independencia de los cambios introducidos por los correctores y adicionadores $^{14}$ de Bravo, el Compendium, tal como lo concibió su autor, contiene unas 14000 entradas, todas, salvo contadas excepciones, latinas. Entre ellas, se encuentra un limitado número de nombres propios (Cocytus; Indus, $i$; Myrtagogus, $i$; Orcus, $i$ ) y construcciones pluriverbales (Atque adeo, Quam primum, Rex facrorum, faepe numero), que pasan casi desapercibidas en la macroestructura del diccionario.

A estas entradas les corresponden otros tantos vocablos, ya que el padre Bartolomé Bravo recoge las distintas acepciones de la voz bajo una sola entrada:

Lumen, inis. lumbre, oluz. lumen alicui preferre. lumen de fuo lumine accendere. Tabulas bene pictas in bono lumine collocare. Ventana. Ædium lumina. Día. Secundo lumine. Ojos. Jumina amittere.

Ouans, antis. El que entraua en Roma con cierta especie de triunfo. In Capitolium ouans arcendit. Alegre. Hortes ouates in ciuitatem irruerunt.

Probo, as, aui, atum. Aprobar. Quis hoc factum non probet. Confilium meu[m] a te probari gaudeo. Hazer experiencia. probare triticu[m]. Mostrar ler verdad, o buena vna cora. Probado. Verre[m] co[n]tra leges pecunias cepirre. Meum ego tibi factum probabo. Tantum contende, quantum probare alicui porsis probauerunt mihi rere, quo minus facerent.

Retineo, es. nui. p[enultima] c[orrepta]. entum. Detener. Retine illum, nifi profectus est. Retener. Pecuniam acceptam retinere, atque fupprimere. Conferuar. Retinere charitatem in alios, aliquem in officio, ius ruum contra aliquem. Entretener, deleytar. Ordo ipfe annaliu[m] nos retinet.

Las acepciones, como se aprecia en los ejemplos anteriores, están deslindadas en función del contexto en que aparece la voz de la entrada, siendo frecuente que el jesuita segoviano indique sus posibles usos metafóricos:

13 Estas listas de voces reemplazan al breve vocabulario de voces tomadas de otros autores latinos de las ediciones anteriores.

14 Además de Pedro de Salas, el Compendium fue corregido por Isidoro López (Madrid, "Ex officina typographi lldephonsi a Lopez", 1785 [en la Biblioteca Nacional de Madrid con la signatura 2/46552] y Alcalá de Henares "Ex officina typographi Petri a Lopez sumptibus suis", 1795 [en la Biblioteca Nacional de Madrid con la signatura 3/52081]), José Carrasco (Barcelona, "In officina Joannis Francisci Piferrer", 1748 [se conserva un ejemplar en la Biblioteca Borja de Sant Cugat del Vallès (Barcelona) con la signatura S-4a-II-9]; Madrid, "Ex officina Joachimi de Ibarra, S. C. M. Typographi. Sumptibus Regiæ Typographorum \& Bibliopolarum Societatis", 1784 [el ejemplar 37-VI-21 de la Biblioteca de la Real Academia Española de la Lengua]; Barcelona, "In officina Joannis Francisci Piferrer, Tipographi Regii, Opus prostat venale, eam regente Joanne Sellent", 1798 [en la Biblioteca de Cataluña con la signatura Res. 1450-8"]; Madrid, "Ex typographia ibarriana", 1799 [en la Biblioteca Nacional de Madrid 3/46768]; Barcelona, "Ex tipographia Sierra, ac Marti", 1826 [en la Biblioteca de Cataluña con la signatura A-42-8 -320 ] y Madrid, "Ex Typographia Ibarriana", 1830 [en la Biblioteca Universitaria de Santiago, L.1403]) y Pedro del Campo Lago (Madrid, "Acad. Socio. Ex Benedicti Cano", 1805 [en la Biblioteca de Cataluña con la signatura A-42-8"-319] y Madrid, "Acad. Socio. Ex Benedicti Cano", 1810 [en la Biblioteca Nacional de Madrid con la signatura U/8457]), pero los cambios lexicográficos son irrelevantes. Por tanto, al igual que ocurre con el Thesaurus, las innovaciones más importantes las introduce el padre Pedro de Salas. 
Angulus, i. p[enultima] c[orrepta]. Erquina. Rincón. Metaph[orice] Lugar recreto. In nullo totius Italiæ angulo.

Fucatus, a, [u]m. Afeytado. fucatus candor. Met[aphorice] Engañoro. Simulatus, \& fucatus homo. In eft in illo oratore naturalis, \& fucatus nitor.

Mollis, e, Blando, [uaue. Infima auricula mollior. mollirsima cera. Metaph[orice] Orationem mollem reddere, ac teneram. molli brachio obiurgare aliquem. Erfe in dolore molliorem. Afeminado.

A diferencia de los diccionarios de Alfonso Fernández de Palencia y Rodrigo Fernández de Santaella, en el Compendium no se llevan a cabo agrupaciones lexemáticas. Los nombres de acción, los diminutivos, los adverbios regulares (-ter) o los escasos participios que se recogen en el repertorio de Bartolomé Bravo tienen, sin excepción, entrada propia:

Afreueratio, onis. Afrmación.

Capella, æ. Cabra pequeña.

Confuetus, a, [u|m. Acoftumbrado. In armis confuetus æuum agit.

Depopulatio, onis. dertruyción.

Feliciter. Difchofamente. Res feliciter euenit [...].

Rumufculus, i. Rumorcillo Imperitorum hominum rumufculos aucupari.

Compárese con el modo en que aparecen algunas de estas informaciones, por ejemplo, en el Vocabularium ecclesiasticum (Sevilla, 1499) de Rodrigo Fernández de Santaella:

Afreuero. ras. p[enultima] p[roducta]. por affirmar. in rermone affumptione. Dende aff eueratio. la affirmación.

Rumor. moris. m[edia] p[roducta]. mar[culini] g[eneris]. por fama. Dende rumurculus. li. diminutiuo. maf[culini] $\mathrm{g}$ [eneris]. por chico rumor o rumorcico o familla q[ue] es chica fama. S. nicholai ${ }^{15}$.

Es más, términos afines fonética y semánticamente tienen entradas diferentes en el Compendium:

Ebrieta, atis. Borrachez.

Ebriolitas, tis. Idem.

Exedra, æ. p[enultima] c[orrepta]. Aula con muchos a[sientos.

Exedria, orum. Idem.

Ignaue. Couardemente.

Ignauiter. Idem.

Libertinus. i. Erclauo horro.

Libertus. i. Idem.

El repertorio latino-español de Bartolomé Bravo se distancia también de diccionarios anteriores en las informaciones que engrosan el artículo lexicográfico. Al igual que en el Thesaurus solo se indica, salvo en raras ocaciones, la categoría gramatical de algunas partículas e interjecciones:

15 Para el Vocabularium ecclesiasticum de Rodrigo Fernández Santaella utilizo el ejemplar de la edición de 1499 conservado en la Biblioteca Nacional de Madrid con la signatura I/1408. 
Cum. aduerbium. Quando. Fuit quoddi tempus, cum homines vagabantur. Que, derpues, que. Dies funt decem, cu[m] te non vidi [...].

De preporitio: de. Rem nullam de mens bonis attigi. Nemo de Graeci. Pecuniam de ruo numerauit. Ecce antem de tranfuerfo. Caerar. De Antonio rum admiratus. Timeo, diffido de illo, feci de tua fententia Vix eft de mea voluntate oncerfum. Ferfus, languen de via. Accipere aliquid de aliquo. Dimicare de imperio.

Hem. Ay. Interiectio.

Más usual es que Bravo señale, si bien no de modo sistemático, la cantidad vocálica de la voz de la entrada:

Admodum. p[enultima] c[orrepta]. Muy. admodum vtile. Litterarum admodum nihil $f$ ciebat.

Efficacitas, atis. p[enultima] c[orrepta]. Eficacia. Tantum habet efficacitatis, vt, \&c.

Hypotheca, æ. p[enultima] p[roducta]. Prenda.

Pacatus, a, [u]m. p[enultima] p[roducta]. Apaciguado, quieto, prouincia pacatirsima.

Peramo, as. p[enultima] clorrepta], aui. atum. Amar mucho.

Singulatim, $\mathrm{p}$ [enultima] $\mathrm{p}$ [roducta]. En particular. Cingilatim vnicuique rerpondere.

La intención didáctica con la que nace el Compendium se aprecia especialmente en la contextualización de la voz latina de la entrada a modo de ejemplo:

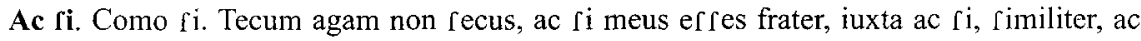
ri, \&c. Pro eo, ac ri concerrum rit, concludis.

Adrcribo, is, pfi. ptum. Efcriuir junto a otra cofa: Diem in epistola adrcribere, ralutem alicui. Atribuyr. Hoc incommodum tibi ad [cibitur [...].

index, cis. El que defcubre, o feñala. Hæc omnia indices detulerunt. Vox index ftulcitiæ vertræ indice. Librorum index.

facrofanctus. a. m. con $\left\lceil\right.$ agrado. Locus racro anctus $^{16}$.

No obstante, son muchos los casos en los que a la entrada latina le sigue exclusivamente el equivalente español. Obsérvese que han desaparecido los enlaces que unían el vocablo latino y el español en los primeros diccionarios bilingües con el español (Alvar Ezquerra, 1990):

Additamentum, ti. añadidura.

Afer. a. m. Cora de África.

Cartellum. i. cartillo.

Columba. æ. Paloma.

Frumentum. i. Trigo.

Fuluus, a. m. Rojo.

Natus, i. Hijo.

Pronunciantum. i. proporición.

Sedenim. Porque.

16 Bartolomé Bravo no especifica el autor del que toma estos ejemplos, pues, como en el Thesaurus, el modelo es Marco Tulio Cicerón, salvo en contadas excepciones:

Balans, tis, Co[a que vala ex Ennio.

Leuipes, dis, p[enultima] cor[repta]. ligera de pies. In Arat[o]. 
Al final del Compendium se recogen a modo de apédice unas 3000 entradas más, bajo el título: "Verba quam plvrima, quæ in ciceronis Scriptus desiderantvr, a varijs scriptoribus collecta"17. En este apéndice, Bartolomé Bravo sigue una estructura similar a la que veíamos en el repertorio lexicográfico con la salvedad de que se especifica el autor del que se toma el vocablo latino de la entrada. Entre los autores citados se encuentran: Virgilio, Terencio, Plinio, Plauto, Quintiliano, Varrón, Horacio, Suetonio, Marcial, Ovidio, etc.

Bafium, ij. Bero. Ter[entius].

Baxea. Calçado de filórofo. Plaut[us].

Egelidus. a m. Medio frío, y caliente. Suet[onius].

Equile, is. p[enultima] p[roducta] caualleriza. Varro.

Hyalus. i. p[enultima] p[roducta]. Vidrio. Virg[ilius].

Imperturbatus, a m. No turbado. Ouid[ius].

præformo, as. Formar antes. Quint[ilianus].

Remirceo, es. Mezclar. Horat[ius].

Zythum, i, Cerueça de trigo o ceuada. Plin[ius].

Son contadas las ocasiones, en las que Bartolomé Bravo no aduce alguna autoridad:

piceus, a m. Cora de pez.

praltes, is. Cantor.

vtto. A qual de dos partes.

vulua, $\mathfrak{x}$. vbre de la baca.

Xiphion, Erpadaña, lirio.

Zelo, as, \& Zelor, aris, tener zelos amar mucho.

Además de la obra de Nizzoli, Bartolomé Bravo debió contar con el diccionario de Ambrogio Calepino o con una edición plurilingüe del Nizzoli, inspirada en el Dictionarium del lexicógrafo bergamasco, pues, como apunta Annamaria Gallina:

il Calepino sará preso a modello da vari lessicografi posteriori, non solo nella parte latina, ma anche in quella plurilingue. L'imitazione sarà più o meno profonda, limitandosi; alcuni all'idea delle traduzioni plurilingui, come il Toscanella, altri copiando senz'altro le traduzioni stesse, como han fatto coloro che aggiusero le traduzioni francesi e spagnole al "Thesaurus" del Nizzoli (1959: 111).

Sirvan como ejemplo algunas de las primeras entradas de la combinación $a b$ de ambos diccionarios ${ }^{18}$ :

17 Este catálogo será sustituido, como ya se ha indicado más arriba, en las ediciones posteriores por una lista de voces sacra y por una de voces hebreas. Estas listas de voces sagradas y hebreas constituyen el punto de partida de las adiciones de Lama Cubero al Vocabularium ecclesiasticum de Rodrigo Fernández de Santaella. Lama Cubero se limita a copiar estas informaciones con cuidado de omitir las voces que ya tenían entrada en el Vocabularium; su intención era evitar que el diccionario de Santaella fuese eclipsado por el enorme éxito del que ya entonces gozaba el Compendium (Medina Guerra, 1996 y 1998a).

18 Para el diccionario de Calepino he utilizado un ejemplar conservado en el Dpto. de Filología Española I de la Universidad de Málaga, catalogado como impreso en Lión por Symphorien Bèrauls Guillaume Roville y Philippi Tingni, 1570. 
Abacus, i. penult[ima] cor[repta] Aparador (Bravo). Abacus, penult[ima] corr[epta] [...] Aparador de la plata o varos [...] (Calepino).

- Abalieno, as, pen[ultima] prod[ucta]. aui. atum. Enagenar [...] (Bravo).

* Abalieno, as, are, pen[ultima] prod[ucta] [...] Enagenar o defapropiar [...] (Calepino) ${ }^{19}$.

- Abauus, i. p[enultima] c[orrepta]. Tercer abuelo (Bravo).

* Abauus, penult [ima] corr[epta] [...] Tercer abuelo (Calepino).

- Abdico, as, p[enultima] c[orrepta]. aui. atum. Desheredar en vida el padre al hijo $[\ldots]$ (Bravo).

* Abdico, as, penult[ima] corr[epta] [...] Empuxar a fuera, echar a fuera desheredar el hijo en la vida $[\ldots]$ (Calepino).

- Abdico. is. xi. ctum. p[enultima] p[roducta]. De echar [...] (Bravo).

* Abdico, penultima producta, cis, xi, ctum [...] Echar atras, o al través [...] (Calepino).

Abdite, p[enultima] c[orrepta]. E[condidamente en efcondido [...] (Bravo).

* Abdite $[\ldots]$ E E condidamente $[\ldots]$ (Calepino).

- Abdo. is. didi. itum. E[conder, encubrir [...] (Bravo).

* Abdo, dis, abdidi, abditum [...] Ef conder [...] (Calepino).

- Abdomen. inis. $\mathrm{p}$ [enultima] $\mathrm{p}$ [roducta]. Vnto, grofura de vientre [...] (Bravo).

* Abdomen, penultima producta [...] A la vntanza o enxundia de animal, como de los puercos $[\ldots]$ (Calepino).

- Abduco, is. p[enultima] p[roducta]. xi. ctum. Lleuar a alguno por fuerça, o apartar de algún lugar $[. .$.$] (Bravo).$

* Abduco. cis. penultima producta [...] Lleuar a alguno por fuerça [...] (Calepino).

- Abeo, is. iui. p[enultima] p[roducta]. itum. p[enultima] c[orrepta]. Ir $\int \mathrm{e}$ [...] (Bravo).

* Abeo, is, abiui, vel abij, abitum [...] Ir a lugar [...] (Calepino).

- Aberratio, onis. Defuio del camino [...] (Bravo).

* Aberratio [...] Apartamie[n]to de camino, errado o defuío [...] (Calepino).

- Aberro. as. aui. atum. Defuiarfe, o apartarfe del camino, yr errado [...] (Bravo).

* Aberro, ras [...] De[uiar[e [...] (Calepino).

La influencia del diccionario de Calepino en el de Bartolomé Bravo es aún más obvia en el apéndice "Verba quam plvriam....". Baste para comprobarlo cotejar las informaciones que el segoviano recoge bajo las primeras 20 entradas con las que da Ambrogio Calepino para estos mismos vocablos:

19 Las entradas Abalienatio, onis, Abazea, vel, Iabazea, orum, Abdera. ce, Abderites, tis, Abderiticus, a. $m$ no están traducidas al español en la edición citada de Lión, 1570. 
- Abactor, oris. p[enultima] p[roducta]. El que hurta ganado, Apul[eius] (Bravo).

* Abactores, qui $\int$ int, qu$\Gamma$ re in dictione Abigeus in $\mathrm{ABIGO}$.

Abigeus [...] Hisp[anicum]. Hurtador de ganado [...] Apuleius \& Paulus lib[ro]. Sentent[iarum]. v. ca[pite] xvIII. Abactorem vocat, Abactores, inquit, funt, qui vnum equum, duas equas, totidemque boues, capras decem aut porcos quinque abegerint (Calepino).

- Abacus, i. p[enultima] c[orrepta]. Tabla para fcriuir, o contar. Perf[ius] Tabla de axedrez. Macrob[ius]. Ef cabelo. Hieron[ymus]. Epicielo del Sol Cæ[lius] (Bravo).

* Abacus, i [...] Hisp[anicum]. Tabla para e[criuir o cortar ; in qua logi $\int \mathrm{t} \int$ numeros, \& mathematici $\int$ uas lineas \& figuras ducunt. Perfius, Nec qui abaci numeros, \& rectos in puluere metas Scit rifif fe vafer $[. .$.$] (Calepino).$

- Abaculus, i. p[enultima] c[orrepta]. Diminit[ivum] ab abaco. Plin[ius] (Bravo).

* Abaculus, diminutiuum eft [...] Hisp[anicum]. Contante para hazer cuenta $\}$ Plin[ius] lib[ro]. 36. cap[ite]. 26 [...] (Calepino).

- Abamita, a. p[enultima] c[orrepta]. Hermana de quarta abuela. Lib[ro]. 3. de Inf titutionum (Bravo).

* Abamita [...] Hisp[anicum]. Hermana del tercero abuelo; Inftit[utionum] lib[ro]. 3 (Calepino).

- Abauia, a. Abuela de mi abuela, lib[ro]. 3. Inftitutionum (Bravo).

* No tiene entrada en Calepino.

- Abuunculus, i. p[enultima] c[orrepta]. Hermano de la tercera abuela, lib[ro]. 3. Inf titut[ionum] (Bravo).

* Abauunculus [...] Hisp[anicum]. Hermano dela tercera abuela\} Inftitut[ionum]. lib[ro]. 3 (Calepino).

- Abax, acis. p[enultima] c[orrepta]. va[ar labonelus (Bravo).

* Abax, acis [...] Hisp[anicum]. Aparador de cofas preciofas? Horum memit labol, in 1. hæred[is]. de legat[ione]. iij [...] (Calepino).

- Abbreuio, as. Abreuiar. Veget[ius] (Bravo).

* Abbreuio, as, are [...] Abbreuiar lo luengo per diuerfos autores librofque difperfa, Imperator in uicte, mediocritatem meam abbreuiare iultifti. \&c. (Calepino).

- Abecedarium, ij. Abecedario, Coeli (Bravo).

* Abecedarius, dicitur rei literariæ tyrunculus, qui adhuc in literis cogno[ cendis hæret. $\alpha \dot{\lambda} \phi \alpha \beta \eta \tau \iota \kappa o ́ s$ Accipitur item pro eo qui aliquid per ordinem alphabeti digerit, vel pro eo quod alphabetico ordine digef tum eft. Vide Cælium libro 14. capite 28. [...] (Calepino).

- Abedo, is. p[enultima] c[orrepta]. edi, efum. $\mathrm{p}$ [enultima] $\mathrm{p}$ [roducta]. Confumir Cornel[ius] Tacit[us] (Bravo).

* Abedo, is, vel abes. abedi. abedere [...] Hisp[anicum].Tragar mucho ; Tac[itus] lib[ro]. 15. Nam exorta vis locultarum abederat quicquid herbidum aut frondof um. (Calepino). 
- Abigeatus. us. p[enultima] p[roducta]. Crimen de hurtar ganado. Marcel[lus]. (Bravo).

* Abigeatus, us [...] Hisp[anicum]. Hurto de ganado\} Marcel[lus]. lib[ro]. 47. Digefto Abigeatus crimen publici iudicij non eft, quia furtum magis elt. (Calepino).

- Abintertato. Sin te[tamento. Afran[ius] (Bravo).

* Abinteftato [...] Hisp[anicum]. Sin teftamento $\}$ [...] Afran[ius]. li[bro]. 29. Dig[estorum]. Quincunque enim abinte tato [ucce $\int$ ferit, locum habent condicilli. Et paulo poft, $\mathrm{Nec}$ inter eft quis fuccedat, dum ab inter tatof uccedat (Calepino).

- Abiho, onis. p[enultima] p[roducta]. EI yrfe. Teren[tius] (Bravo).

* No lo he encontrado en Calepino.

- Abiugo, as. p[enultima] c[orrepta]. Quitar del iugo. Apartar. Pacu[uius] (Bravo).

* Abiugo [...] Hispa[nicum]. Apartar o defunir vno de otro yt, Abiugare bouem, hoc eft, a pari difiungere [...] Pacuuius. Quæ res te ab [tabulis abiugat? Adiugo, de quo infra (Calepino).

- Ablacto, as. Der tetar (Bravo).

* Ablacto, as [...] Deftetar\} Virg[ilius] dixit à lacte depello [...] (Calepino).

- Ablaqueo, as, aui, atum. Efcauar los árboles.Colum[ella]. Vnde Ablaqueatio. (Bravo).

* Ablaqueo [...] Hisp[anicum]. E[cauar o alimpiar los árboles \} Col[umella] lib[ro]. 3. [...] Hinc Ablaqueatio, [...] Hisp[anicum]. Aquella obra de efcauar los árboles (Calepino).

- Ablatiuus. i. p[enultima] p[roducta]. Ablatiuo caro. Quint[iliano] (Bravo).

* Ablatiuus cafus, Grammaticis dicitur, qui $\int$ extus eft ca〔us apud Latinos, \& alio nomine, tefte Prifciano. Co[m]paratiuus appellatur, eo quod per eum non minus co[m]paratio fignificetur, quam ablatio [...] Ablatiuu[m] Græci no[n] habe[n]t: vocatur tamen a Quintiliano [...] (Calepino).

- Ablepra, æe, p[enultima] c[orrepta]. Ceguera de ánimo. Sueton[ius] (Bravo).

* Ablepfa. Latine cæcitas, \& inconfideratia [...] Suet[onius] in Claudio [...] Hisp[anicum]. La ceguedad, o poco miramiento) (Calepino).

- Abligurio, guris. p[enultima] p[roducta]. iui, itum. Galtar en golof inas. Terent[ius] (Bravo).

* Abligurio, ris [...] Galtar la hazienda en golo[inas $\}$ Ter[entius] in Eunuch[o] [...] (Calepino).

- Abludo, is, fi, rum. $\mathrm{p}$ [enultima] $\mathrm{p}$ [roducta]. Diferenciarfe. Horat[io] (Bravo).

* Abludo, pen[ultima] pro[ducta] [...] Hisp[anicum] Discordar? Horat[io] [...] (Calepino).

Es más, en la edición vallisoletana de 1671, el nombre de Calepino pasa a ocupar un lugar relevante en la portada: Compendium latino-hispanum utriusque lingua veluti lumen, quo Calepini, Thesauri Enrici Stephani, Antonii Nebrissensis, Nizolii, P. Bartholomaei Bravo atque omnium optimae notae authorum labores et lucubrariones perspicua brevitate continentur. Con este mismo título se conocen, como mínimo, las siguientes ediciones: 
1) Alcalá de Henares, Apud Franciscum Garcia Fernandez Typographum Vniversitatis. Acosta de Iuan Vicente Mercader de libros frontero de S. Felipe, $1672^{20}$.

2) Madrid, "Ex Tipographia Bernardi à Villa Diego, Regis, ac Diuini Officij Typograhi. Sumptibus Floridi Assisson", 169521.

3) Amberes, "Apud Henricum, \& Cornelium Verdussen. A costa de Francisco Lasso, Mercader de Libros", $1724^{22}$.

4) Amberes, "Apud Henricum, \& Cornelium Verdussen. A costa de Juan Oliveras, mercader de libros, heredero de Francisco Laso", $1732^{23}$

5) Amberes "Apud Henricum, \& Cornelium Verdussen. A costa de Luis Correa, Mercader de libros", $1740^{24}$.

6) Pamplona, "Ex Typographia latina D. M. A. Domech suis expensis: prostatque sua Bibliotheca", 1761.

La presencia del nombre de Calepino en la portada y en el lomo de ediciones como las mencionadas explica que el Compendium fuese conocido popularmente como el Calepino de Salas $^{25}$. Evidentemente, no podemos pasar por alto que, como es sabido, la parte española del diccionario de Calepino tiene mucho que ver con la obra de Antonio de Nebrija ${ }^{26}$ y que, por tanto, al igual que en el Thesaurus (Medina Guerra, 1998b), su influencia -ya sea direnta o indirectamente a través del lexicógrafo italiano- se deja sentir en el Compendium. De ahi, que a diferencia de otros autores, como Rodrigo Fernández de Santaella, Diego Jiménez Arias o Alonso Sánchez de la Ballesta (Medina Guerra, 1995-1996), el jesuita segoviano se nos presente como un eslabón más de la cadena lexicográfica iniciada por Nebrija un siglo antes, pues, como él, huye de las informaciones enciclopédicas y opta por la simplicidad de las equivalencias, aunque superando uno de los aspectos más criticados del maestro de Lebrija: la falta de citas-ejemplos.

\section{Referencias bibliográficas}

Ajo González y Sáinz de Zúñiga, C. M.“. (1957-1972): Historia de las universidades hispánicas. Origenes y desarrollo desde su aparición a nuestros dias, Ávila, Senén Martín, 8 vols.

Alvar Ezquerra, M. (1990): "La confección de diccionarios", Voz y Letras, I-1, pág. 47-76.

20 Se conservan ejemplares en la Biblioteca Nacional de Madrid (2/3802 I y $2 / 46581$ ) y en la Biblioteca de Filología de la Universidad Complutense de Madrid (30125).

21 El único ejemplar que conozco se halla en una biblioteca particular en Madrid.

22 He manejado el ejemplar de la Biblioteca Nacional de Madrid (3/42294).

23 Ejemplares en la Biblioteca Nacional de Madrid (2/23008) y en la Biblioteca Universitaria de Salamanca (32.968).

24 En la Biblioteca Nacional de Madrid se conserva un ejemplar con la signatura 2/69393.

25 El término calepino no sería en este caso, como se ha creído hasta ahora, tanto sinónimo de diccionario, como testimonio de una verdad: el diccionario de Calepino es en muchos aspectos la fuente del Compendium incluso antes de ser aumentado por Pedro de Salas.

26 "No si conosce il nome di colui che aggiunse la prima volta la traduzione spagnuola, ma, giè s'e visto che, chiunque fosse, attinse senza scrupoli all'opera fondamentale della lessicografia spagnuola: il Dictionarium de Antonio Nebrija, e tutte le edizioni posteriori conserveranno inalterato il primitivo testo, attraverso più o meno numerosi errori de stampa" (Gallina, 1959: 111). 
Baeza y González, T. (1877): Apuntes biográficos de escritores segovianos, Segovia, Imp. de la viuda de Alba y Santiuste.

Cooper, L. (1962): "Plagiarism in Spanish Dictionaries of the xvith. centuries", Hispania, XLV-4, págs. 717-720.

Gallina, A. (1959): Contributi alla storia della lessicografia italo-spagnola dei secoli XVI e XVII, Florencia, Leo S. Olschi.

Medina Guerra, A. M." (1995-1996): "El latín y el español en los diccionarios de los siglos XVI y XVII", Revista de Lexicografia, II, págs. 61-72.

Medina Guerra, A. M." (1996): "Epígonos de Rodrigo Fernández de Santaella". En Luque Durán, J. de D. y A. Pamies Bertrán (eds.): Segundas jornadas sobre el estudio y la enseñanza del léxico (Granada, abril 1995), Granada, Metodo Ediciones, págs. 121-130.

Medina Guerra, A. M." (1997): "Las fuentes del Thesaurus de Bartolomé Bravo". En Molina Redondo, J. A. de y J. de D. Luque Durán (eds.): Estudios de lingüistica general (III), Granada, Granada Lingüística y Método Ediciones, págs. 315-322.

Medina Guerra, A. M." (1998a): Las ediciones del Vocabularium seu Lexicon ecclesiasticum de Rodrigo Fernández de Santaella y Diego Jiménez Arias (1499-1798), Málaga, Sevicio de Publicaciones de la Universidad.

Medina Guerra, A. M. (1998b): "La labor lexicográfica del padre Pedro de Salas”. En Luque Durán, J. de D. y F. J. Manjón Pozas (eds.), Teoria y práctica de la lexicología (IV Jornadas Internacionales sobre Estudio y Enseñanza del Léxico), Granada, Método Ediciones, págs. 175-184.

Palau y Dulcet, A. (1948-1977, 2." ed. revisada y añadida por Agustín Palau Claveras): Manual del librero hispanoamericano. Bibliografia general española e hispanoamericana desde la invención de la imprenta hasta nuestros tiempos con el valor comercial de los impresores descritos, BarceIona, Librería Anticuaria de A. Palau, $28 \mathrm{v}$.

Vergara y Martín, G. M. (1903): Ensayo de una colección bibliográfica-biográfica de noticias referentes a la provincia de Segovia, Guadalajara, Taller tipográfico del Colegio de Huérfanos de la Guerra. 